La Revue

des Droits

de l'Homme

\section{La Revue des droits de l'homme}

Revue du Centre de recherches et d'études sur les droits fondamentaux

$10 \mid 2016$

Revue des droits de l'homme - $\mathrm{N}^{\circ} 10$

\title{
Entre alerter et ne pas risquer de s'auto- incriminer, leur cœur balance (groupe militant Breaking the silence/ Israël-Palestine)
}

\section{Rebecca Mignot-Mahdavi}

\section{OpenEdition}

Édition électronique

URL : http://journals.openedition.org/revdh/2405

DOI : $10.4000 /$ revdh.2405

ISSN : 2264-119X

Éditeur

Centre de recherches et d'études sur les droits fondamentaux

Référence électronique

Rebecca Mignot-Mahdavi, «Entre alerter et ne pas risquer de s'auto-incriminer, leur cœur balance (groupe militant Breaking the silence/ Israël-Palestine) », La Revue des droits de l'homme [En ligne], 10 2016, mis en ligne le 07 juillet 2016, consulté le 22 juillet 2020. URL : http://journals.openedition.org/ revdh/2405; DOI : https://doi.org/10.4000/revdh.2405

Ce document a été généré automatiquement le 22 juillet 2020.

Tous droits réservés 


\title{
Entre alerter et ne pas risquer de s'auto-incriminer, leur cœur balance (groupe militant Breaking the silence/ Israël-Palestine)
}

\author{
Rebecca Mignot-Mahdavi
}

Shovrei Shitka, ou les briseurs de silence, en hébreu : c'est ainsi que sont désignés les membres de l'association «Breaking the silence », auteurs de l'ouvrage «Le livre noir de l'occupation israélienne »: Les soldats racontent, regroupant cent quarante-cinq témoignages sur les exactions commises en territoires palestiniens occupés par l'armée israélienne ${ }^{1}$. En juin 2004, une soixantaine de vétérans des forces armées israéliennes présentaient publiquement des photographies et témoignages écrits retraçant leur service militaire effectué à Hébron et en Cisjordanie occupée. Cet événement marquait la naissance de l'association "Breaking the silence». Les cent quarante-cinq témoignages publiés ne sont qu'une bribe de la totalité des témoignages, plus de sept cent, récoltés par le groupe militant. Tous, assurent-ils, ont été vérifiés et recueillis par des vétérans de l'armée israélienne. L'ouvrage précise que les récits émanent de témoins oculaires et sont retranscrits mot pour mot. Par ailleurs, le travail de l'association est gouverné par le principe de confidentialité. Il est aisément concevable que l'anonymat des témoignages soit une condition de leur existence. En l'absence d'un tel principe, il est fort probable que peu de récits soient livrés, face à un État qui considère les auteurs de ces témoignages comme des traitres ${ }^{2}$. Aussi cet anonymat trouve-t-il une explication dans la crainte qu'éprouveraient les auteurs des témoignages, dans le cas contraire, de voir leur responsabilité pénale engagée. En effet, les auteurs des récits contenus dans l'ouvrage qui nous intéresse retracent les exactions qu'ils ont eux-mêmes commises, de leur plein gré ou sur les ordres de leurs supérieurs hiérarchiques. Accumulées, ces histoires sont autant de liens tissés qui révèlent plus globalement les rouages de l'appareil sécuritaire et du système militaire israélien mis en œuvre dans les territoires occupés. 
2 Les opérations menées semblent pouvoir être constitutives de crimes internationaux les plus graves, dont la poursuite et le jugement pourraient relever de la compétence de la Cour pénale internationale : crime de guerre, crime contre l'humanité3. Les exactions relatées par les auteurs des témoignages de «Breaking the Silence» sont susceptibles de relever de la compétence de la Cour pénale internationale. En tout état de cause, en choisissant d'ouvrir les portes de leur quotidien militaire en territoires palestiniens occupés, les auteurs de ces récits acceptent de courir le risque d'être eux-mêmes poursuivis. La question soulevée par cette situation est celle de savoir si une figure de lanceur d'alerte doit et peut être saisie par le droit international pénal. Avant de se pencher sur la possibilité de qualifier ces individus de lanceurs d'alerte et sur la possibilité, pour le droit international pénal existant, de s'en saisir, afin de mettre en œuvre un cadre juridique protecteur pour ces lanceurs d'alerte, une question préalable doit être envisagée : la Cour pénale internationale, dans le cadre de laquelle s'inscrivent les présentes réflexions, peut-elle être compétente pour connaître des crimes commis sur le territoire palestinien par des soldats israéliens? Des évolutions récentes, intervenues au terme de l'année 2014 qui a vu l'éclatement de nouvelles frappes et l'intensification des violences dans cette région du monde, permettent de formuler une réponse affirmative à cette question.

3 Le 1er janvier 2015, le Greffier de la Cour pénale internationale (CPI), Herman von Hebel, recevait une déclaration déposée par le gouvernement palestinien déclarant son acceptation de la compétence de la CPI à partir du 13 juin 2014, en vertu de l'article 12 (3) du Statut de Rome. Cet article offre aux États non parties au Statut de Rome, la possibilité d'accepter la compétence de la CPI à leur égard. L'acceptation de la compétence de la CPI ne correspond pas à une adhésion au Statut de Rome. Alors, pour aller encore plus loin, le 2 janvier 2015, la Palestine a transmis à l'Organisation des Nations Unies (ONU), les documents relatifs à son accession au Statut de Rome. Après examen de ces documents, le mercredi 7 janvier 2015, l'ONU a accepté la demande d'adhésion de la Palestine à la Cour pénale internationale ${ }^{4}$. Or, l'article 125 du Statut de Rome ne mentionne que les États comme pouvant être parties au Statut et ne fait état d'aucune autre entité juridique comme pouvant être partie au Statut. Cela vaut donc reconnaissance à la Palestine du statut d'État au sein de la CPI et a vraisemblablement été permis, ou à tout le moins impulsé, par la décision de l'Assemblée générale des Nations Unies d'accorder à la Palestine le statut d'État non membre observateur auprès de l'ONU le 29 novembre 2012.

4 Le vendredi 16 janvier 2015, la Procureure de la Cour pénale internationale (CPI), Mme Fatou Bensouda, ouvrait un examen préliminaire de la situation en Palestine. Il lui reviendra de décider si les critères établis par le Statut de Rome pour l'ouverture d'une enquête sont réunis. ${ }^{5}$. Peu importe l'issu de cet examen, la CPI est désormais compétente pour connaître de la situation en Palestine pour la période qui s'écoule depuis le 13 juin 2014, et donc potentiellement des faits relatés par les témoignages qui nous intéressent. Plus encore, il est envisageable que ces témoignages attirent l'attention de la Cour.

5 Il ne sera pas ici envisagé la question de la qualification du conflit. Cette interrogation n'est pas indispensable à notre réflexion. En effet, si les crimes de guerre relevant de la compétence de la CPI requièrent un travail de qualification du conflit en conflit armé, tel n'est pas le cas pour les crimes contre l'humanité. 
6 Les soldats israéliens qui font état des exactions commises par les forces armées auxquelles ils appartenaient peuvent-ils être qualifiés de lanceurs d'alerte? Le droit pénal international a-t-il intérêt, et est-il doté des outils nécessaires pour se saisir de la figure de lanceur d'alerte?

7 Nous commencerons par envisager la possibilité de qualifier les soldats lanceurs d'alerte, ainsi que la nécessité de les protéger en tant que tels pour que la justice pénale internationale puisse fonctionner. Ensuite, nous partirons à la recherche d'un régime protecteur dans le droit international pénal existant de ces soldats lanceurs d'alerte et nous nous interrogerons sur la capacité d'un tel régime à protéger effectivement ces lanceurs d'alerte.

\section{I- La nécessité de protéger les soldats lanceurs d'alerte, condition du bon fonctionnement de la justice pénale internationale}

8 Afin de s'assurer de la pertinence de la question étudiée, il convient dans un premier temps de vérifier que la CPI est susceptible d'être matériellement compétente pour connaître des actes révélés par les témoins. Démontrer que les faits relatés peuvent être constitutifs des incriminations prévues par le Statut de Rome est une étape nécessaire à la prise de conscience du risque que les témoins en cause acceptent de courir : l'auto-incrimination. Nous procéderons alors à la qualification des individus auteurs des témoignages recueillis dans l'ouvrage du Livre noir de l'occupation israélienne en lanceurs d'alerte.

\section{A- La compétence de la CPI à l'égard des actes révélés par les témoins}

9 Il convient de se demander si les actes que les auteurs des témoignages ont commis sont effectivement incriminables par la CPI et s'il existe des modes de responsabilité correspondant à la forme de participation des témoins dans l'accomplissement de ces actes. Pour le savoir, nous proposons d'effectuer un travail de qualification des infractions sur le fondement de quelques témoignages tirés de l'ouvrage « Le livre noir de l'occupation israélienne ». Le choix de ces témoignages s'est effectué en fonction de leur représentativité des faits relatés dans de multiples récits. Bien sûr, cet exercice de qualification est indicatif puisqu'il reviendrait à la Procureure de la CPI de vérifier la fiabilité des éléments de preuve fournis par les témoignages et d'effectuer ce travail de qualification.

10 L'article 7 du Statut de Rome incrimine en tant que crimes contre l'humanité l'un quelconque des actes de meurtre, extermination, réduction en esclavage, déportation ou transfert forcé de population, emprisonnement ou autre forme de privation grave de liberté physique en violation des dispositions fondamentales du droit international, torture, viol, esclavage sexuel, prostitution forcée, grossesse forcée, stérilisation forcée ou toute autre forme de violence sexuelle de gravité comparable, disparitions forcées de personnes, crime d'apartheid et autres actes inhumains de caractère analogue causant intentionnellement de grandes souffrances ou des atteintes graves à l'intégrité physique ou à la santé physique ou mentale, lorsque cet acte est commis dans le cadre 
d'une attaque généralisée ou systématique lancée contre toute population civile et en connaissance de cette attaque. Pour qu'un crime contre l'humanité soit constitué, il faut donc, à la fois, démontrer l'existence d'une attaque généralisée ou systématique contre la population civile ${ }^{6}$ et la commission d'actes dans le cadre de cette attaque, qui seront caractérisés suivant ce que l'on peut appeler des «sous-qualifications ». Ainsi, pour qu'un crime contre l'humanité soit caractérisé, la Procureure devra d'abord qualifier l'existence d'une attaqué généralisée ou systématique contre la population civile. Aussi, comme mentionné plus avant, il faudra dans un premier temps qualifier un conflit armé pour conclure à la caractérisation d'un crime de guerre. Puis, il s'agira de vérifier que les actes isolés retranscrits peuvent constituer une des sousqualifications du crime contre l'humanité. Prenons l'exemple du Témoignage $n^{\circ} 3$, dont un extrait est ci-après retranscrit :

« Unité : Brigade KFIR, Lieu : région de Naplouse, Année 2009.

Question: Pendant votre service dans les territoires, qu'est-ce qui vous a le plus secoué?

Réponse : Les fouilles que nous avons faites à Hares. C'est la goutte d'eau qui a fait déborder le vase. Ils nous ont dit qu'il fallait fouiller soixante maisons. J'ai pensé qu'ils devaient avoir des infos des services de renseignements, j'essayais de me justifier la chose.

$\mathrm{Q}$ : C'était le jour ou la nuit?

$\mathrm{R}$ : La nuit. Nous sommes allés de maison en maison frapper à la porte de familles à deux heures du matin. Ils étaient terrorisés, les filles se faisaient pipi dessus de peur. On cognait aux portes avec un sentiment de "on va leur montrer ", c'était hallucinant. On entrait dans la maison et on mettait tout sens dessus dessous. Quelle est la procédure ? Rassembler la famille dans une pièce, placer un garde à la porte, lui ordonner de braquer son arme sur eux puis fouiller toute la maison. Tous ceux entre seize et vingt-neuf ans, on devait les emmener menottés, les yeux bandés. Les soldats criaient sur les personnes âgées, l'une d'entre elles a fait une crise d'épilepsie. Vous vous rappelez autre chose de ce soir-là ? Qui m’a dérangé ? Un petit truc, mais ça m'a gêné. Une maison de civils qu'ils ont juste démolie ».

L'article 7, paragraphe k) du Statut de Rome incrimine en tant que crime contre l'humanité les «autres actes inhumains de caractère analogue causant intentionnellement de grandes souffrances ou des atteintes graves à l'intégrité physique ou à la santé physique ou mentale ». L'article 8 2) b) ii incrimine en tant que crime de guerre le fait de diriger intentionnellement des attaques contre des biens de caractère civil, c'est-à-dire des biens qui ne sont pas des objectifs militaires. Enfin, l'article 8 2) b) 23) incrimine le fait d'utiliser la présence d'un civil ou d'une autre personne protégée pour éviter que certains points, zones ou forces militaires ne soient la cible d'opérations militaires. Les faits retranscrits dans le témoignage $n^{\circ} 3$ sont susceptibles de remplir les éléments de certaines sous-qualifications des crimes de guerre et contre l'humanité prévues par le Statut de Rome.

Par ailleurs, certains témoignages évoquent l'envoi de personnes civiles sur le terrain pour désamorcer des explosifs. La procédure employée est identifiée par les soldats comme la "procédure de voisinage ». Ils la qualifient de "procédure de base", « employée beaucoup, tout le temps ». Le témoin $\mathrm{n}^{\circ} 7$ raconte $^{7}$ :

«Question : Vous l'avez vraiment utilisé comme bouclier humain?

Réponse: Oui un bouclier humain dans le sens où s'il y avait quelque chose de

dangereux...

$\mathrm{Q}$ : Pourtant vous avez un robot.

$\mathrm{R}$ : Mais il faut un temps infini pour le mettre en route et l'amener sur place.

$\mathrm{Q}$ : Vous aviez peur? 
R: Quoi ? Sans doute. Avec les explosifs improvisés, la moindre erreur est extrêmement dangereuse. Si vous avez un téléphone sur vous, c'est extrêmement dangereux. Quand on sait à quel point c'est dangereux, on n'a pas envie de s'en approcher. Alors souvent on utilisait... ».

De nombreux témoins racontent avoir quotidiennement causé la mort d'individus dont rien ne démontrait la participation active aux hostilités, souvent sur ordre des supérieurs hiérarchiques, mais aussi de leur plein gré8. Or, l'article 7, paragraphe 1) prévoit l'incrimination de crime contre l'humanité par meurtre. Pour être caractérisée, l'infraction exige d'abord que l'auteur ait causé la mort d'une ou plusieurs personnes, dans le cadre d'une attaque généralisée ou systématique contre la population civile. Ensuite, il faut avoir des éléments démontrant que l'auteur savait que ce comportement faisait partie d'une attaque généralisée ou systématique dirigée contre une population civile ou entendait qu'il en fasse partie.

Il est important de souligner que tous ces témoins ne seront pas susceptibles de voir leur responsabilité engagée en tant qu'auteurs directs. Néanmoins, il existe une multiplicité de modes de responsabilité à l'infraction en droit international pénal, dont certains sont parfois très peu exigeants quant au niveau de participation et d'implication requis9. La complicité par omission a par exemple été retenue par le tribunal pénal international pour le Rwanda dans le jugement portant condamnation de Vincent Rutaganira ${ }^{10}$ pour extermination en tant que crime contre l'humanité, pour avoir entre le 14 et le 17 avril 1994 environ participé, par omission, aux massacres survenus à l'église de Mubuga, qui ont fait des milliers de morts et de nombreux blessés parmi les réfugiés tutsi qui s'y trouvaient.

La CPI pourrait vraisemblablement être compétente pour connaître des actes perpétrés par les témoins. En révélant les exactions qu'ils ont commises au sein de l'armée israélienne, les témoins de "Breaking the Silence» prennent le risque de s'autoincriminer.

\section{B- La qualification des soldats témoins risquant de s'auto-incriminer en lanceurs d'alerte}

« Voilà pourquoi la droite dure israélienne voit en ces témoins, qui mettent à jour sa vraie nature à la face du monde, des traîtres. Pour ma part, ces hommes et ces femmes, soldats d'une armée dans les rangs de laquelle j'ai combattu et à laquelle j'ai appartenu pendant plus d'un tiers de siècle, affichent les plus hautes vertus de citoyen, au premier rang desquelles vient le courage ${ }^{11}$. C'est ainsi que Zeev Sternhell, professeur émérite de sciences politiques à l'université hébraïque de Jérusalem ouvre en sa préface le Livre noir de l'occupation israélienne. Pour reprendre les définitions posées par Danièle Lochak, le lanceur d'alerte dans son acception étroite est celui qui, dans son champ professionnel, constate l'existence d'un danger grave et collectif, et qui, après avoir en vain cherché à faire intervenir ceux qui ont compétence pour le prendre en charge, entre dans des stratégies de résistance au risque de s'attirer des mesures de rétorsion ${ }^{12}$. L'alerte a lieu dans le contexte d'une relation de travail. Dans un sens plus large, le terme peut s'appliquer à « toute personne ou groupe qui rompt le silence pour signaler, dévoiler ou dénoncer des faits, passés, actuels ou à venir, de nature à violer un cadre légal ou réglementaire ou entrant en conflit avec le bien commun ou l'intérêt général » 
ou encore «toute personne soucieuse qui tire la sonnette d'alarme afin de faire cesser des agissements pouvant représenter un risque pour autrui $»^{13}$.

Ainsi, pour qualifier un individu de lanceur d'alerte, il convient de s'attacher, d'une part, à la nature des faits dénoncés et au contexte dans lequel cette dénonciation intervient, et d'autre part, à la posture dans laquelle se place de lanceur d'alerte lorsqu'il la lance, vis-à-vis de l'institution à laquelle il appartient, mais pas seulement. Non seulement la situation qui nous intéresse correspond à l'acception étroite du lanceur d'alerte, mais aussi répond-elle aux conditions de qualification du lanceur d'alerte dans son acception étroite. En effet, lorsque l'on s'attarde sur la nature des faits dénoncés ici, outre le fait qu'ils constituent des comportements nuisibles à l'intérêt général ou encore diverses atteintes potentielles aux droits et libertés - comme le prévoit la conception large du lanceur d'alerte - ils sont aussi plus précisément des faits potentiellement constitutifs de crimes comme la conception plus étroite le prévoit. Quant au contexte, défini comme le contexte de travail si l'on s'en tient à la conception étroite du lanceur d'alerte, il s'agit dans notre cas du contexte professionnel de l'armée. Lorsque l'on s'attarde ensuite sur la posture délicate dans laquelle se place le lanceur d'alerte lorsqu'il la lance, qui nous semble être un dénominateur commun des lanceurs d'alerte, notons que les soldats qui ont témoigné se placent non seulement dans une posture délicate vis-à-vis de l'institution à laquelle ils appartiennent, de l'État duquel ils sont ressortissants, mais aussi vis-à-vis d'une juridiction internationale à vocation universelle qui peut avoir compétence pour les poursuivre et les juger. En tout état de cause les soldats témoins risquant de s'auto-incriminer par la divulgation de leur récit peuvent être qualifiés de lanceurs d'alerte.

Le témoignage étant la reine des preuves en droit international pénal, et notamment à la Cour pénale internationale, il est indispensable de protéger les témoins - y compris les témoins auteurs de faits susceptibles de conduire à une incrimination - afin de préserver les informations qu'ils délivrent.

\section{II - La nécessaire recherche d'un régime protecteur des soldats lanceurs d'alerte}

19 Le témoignage en droit international pénal revêt une double caractéristique : il est à la fois fragile et indispensable. Dans le cas des témoins risquant de s'auto-incriminer, la fragilité du témoignage est d'autant plus fort que les risques pris sont plus nombreux. En effet, non seulement le témoin craint les représailles mais aussi appréhende-t-il les poursuites judiciaires qui pourront être ouvertes à son encontre. Aussi, l'importance du témoignage est-elle substantielle dans le cas du témoin lanceur d'alerte. Dans ce cas, le témoin délivre des informations sur le fonctionnement intérieur d'un système auquel il est très difficile d'avoir accès. Ainsi, il convient d'identifier ce qui peut être considéré comme un régime protecteur pour les individus qui témoignent alors même que leur témoignage pourrait éventuellement être retourné contre eux. 


\section{A- La nécessité de protection des témoignages - preuve reine à la $\mathrm{CPI}$ - et de leurs auteurs}

Les procédures pénales internationales dépendent considérablement des témoignages ${ }^{14}$. La particulière importance de la preuve par témoignage pour le bon fonctionnement de la justice pénale internationale apparaît au sein même du statut de Rome qui, en son article 69 intitulé «Preuve » s'intéresse en premier lieu et dans ses quatre premiers alinéas aux témoignages ${ }^{15}$. Une des grandes difficultés que rencontre la justice pénale internationale est incontestablement l'accès à des preuves permettant d'établir l'innocence ou la culpabilité des personnes mises en cause. Surtout, lorsque les faits se sont déroulés en temps de guerre, ou de troubles et tensions internes très violents, ou encore dans des pays dans lesquels l'écrit n'occupe pas une place prédominante dans l'organisation de l'État, les juges internationaux se fondent en grande partie sur ce que relatent des témoins visuels, oculaires et/ou des victimes des exactions commises, et/ou, mais plus rarement car soumis au risque de s'auto-incriminer, comme dans notre cas, des témoins qui ont potentiellement également commis des infractions. La collecte, ainsi que l'appréciation de ce ces témoignages, revêt une certaine complexité : les auteurs de ces témoignages peuvent légitimement craindre des représailles, mais aussi dans le cas qui nous intéresse, de s'auto-incriminer ${ }^{16}$.

Certaines affaires devant la Cour pénale internationale sont révélatrices à la fois de l'importance et de la fragilité du mode de preuve par témoignage en droit international pénal. Les affaires kényanes, «le Procureur c. William Ruto et Joshua Arap Sang » et «Le Procureur c. Uhuru Kenyatta » sont éloquentes sur ce point. Après la clôture de la procédure à l'encontre du président du Kenya, Uhuru Kenyatta, le 13 mars 2015, pour manque de preuves ${ }^{17}, 2016$ a marqué la clôture de celle de William Ruto, vice-président du Kenya, et de Joshua Arap Sang. Lors de la procédure conduite à leur encontre, seize des trente-deux témoins prévus par l'accusation ont, soit refusé de se rendre à la CPI, soit sont revenus sur leurs dépositions initiales, lorsqu'ils se sont exprimés devant la Cour. En conséquence du manque de moyens à charge, le 5 avril 2016, la Chambre de première instance $\mathrm{V}(\mathrm{A})$ de la CPI a décidé, à la majorité de ses membres, Mme la juge Olga Herrera Carbuccia étant en désaccord ${ }^{18}$, qu'il devait être mis fin à l'affaire concernant William Samoei Ruto et Joshua Arap Sang ${ }^{19}$. Ces affaires témoignent de deux enjeux majeurs auxquels la justice pénale internationale est confrontée. D’abord, elles révèlent la fragilité des témoins et des informations qu'ils détiennent. Ensuite et en conséquence, elles font état de la fragilité des poursuites en l'absence de suffisamment de témoignages. S'il n'est en aucun cas déplorable que la Cour mette fin à la procédure lorsque les preuves ne sont pas suffisantes, ces affaires alertent sur la protection particulière dont doivent bénéficier les témoins, au risque de voir les procédures menées par la CPI « tuées dans l'œuf ».

Dans le cas des témoins lanceurs d'alerte, risquant de s'auto-incriminer, le risque pris est multiple. Une protection particulière de ces témoins, en complément de la protection normalement accordée aux témoins par la CPI est à envisager. 


\section{B - Le droit de ne pas s'auto-incriminer : un régime protecteur à perfectionner des témoins lanceurs d'alerte}$$
\text { de procédure et de preuve de la CPI }
$$

Elle prévoit qu'un témoin peut refuser de faire toute déclaration qui risquerait de l'incriminer. Alternativement, la Chambre peut ordonner au témoin de répondre à la question ou aux questions après lui avoir garanti que les éléments de preuve contenus dans sa déposition resteront confidentiels et ne seront pas révélés au public ou à un État et ne seront pas utilisés directement ou indirectement contre lui dans le cadre de poursuites ultérieures devant la Cour. Afin de rendre effective la garantie offerte au témoin risquant de s'auto-incriminer, la Chambre peut avoir recours à différents outils prévus par la Règle $n^{\circ} 74$. Elle peut, par exemple, ordonner que la déposition se fasse à huis-clos, ordonner que l'identité du témoin et le contenu de sa déposition ne seront divulgués d'aucune façon, sous peine de sanction, ou encore ordonner la mise sous scellés des procès-verbaux. Aussi, dans le cas où la question d'auto-incrimination d'un témoin se poserait en cours d'instance, la Chambre aurait la possibilité de suspendre l'audition du témoin et de lui donner la possibilité d'obtenir un avis juridique aux fins de l'application du régime de protection de la Règle 74 .

Les témoins qui risquent de s'auto-incriminer sont des témoins sur lesquels il pèse des soupçons d'avoir participé à la commission des faits dont est saisie la cour, sans pour autant que ces soupçons soient suffisamment avérés pour justifier une mise en examen. C'est ce qui a fait dire à certains auteurs que le témoin assisté est en réalité « un suspect qui le droit de se défendre ", ou encore un "para-suspect ». Ils insistent de façon croissante sur la nécessité pour ces témoins de bénéficier de l'assistance d'un conseil ${ }^{21}$. Or, à l'instar des autres tribunaux pénaux internationaux, aucun des textes fondamentaux de la CPI ne prévoit le droit au conseil au bénéfice des témoins susceptibles de s'auto-incriminer. Les juges, qui mesurent eux aussi la nécessité de leur accorder l'assistance d'un conseil, adoptent une démarche proactive et tendent à faire du droit au conseil du témoin la règle dès lors qu'il y a un risque d'auto-incrimination, depuis une décision orale du 28 janvier 2009 dans l'affaire Lubanga ${ }^{22}$.

Il semble que la saisine par le droit international pénal de la figure du lanceur d'alerte comme statut des témoins qui risquent de s'auto-incriminer puisse mettre encore davantage l'accent sur la nécessité d'améliorer leur protection et la rendre plus effective et, partant, de soulever les obstacles au recueil de preuves que constituent les craintes de ceux qui risquent de s'auto-incriminer. Il semble, en effet, que la dénomination de lanceur d'alerte souligne la particulière vulnérabilité de celui qui 
émet l'alerte et la nécessité de le protéger, mais aussi la particulière importance des faits initialement secrets ou difficilement saisissables qu'il révèle.

\section{NOTES}

1. Breaking the silence, Le livre noir de l'occupation israélienne : Les soldats racontent, Éditions Autrement, Paris, 2013, 396 p.

2. Z. Sternhell, Préface «Les Briseurs de glace», in Le Livre noir de l'occupation israélienne, Éditions Autrement, Paris, Octobre 2013 ; voir aussi, « Israël : ces " nouveaux traîtres » à la tête des ONG de gauche », Le Point, par Danièle Kriegel, 20 décembre 2015, http://www.lepoint.fr/ monde/israel-ces-nouveaux-traitres-a-la-tete-des-ong-de-gauche-20-12-2015-2004104_24.php.

3. Human Rights Watch, Precisely Wrong. Gaza Civilians Killed by Israeli Drone-Launched Missiles, June 2009; Report of the United Nations Independent Commission of Inquiry on the 2014 Gaza Conflict, A/HRC/29/52, 24 June 2015: "In relation to this latest round of violence, which resulted in an unprecedented number of casualties, the commission was able to gather substantial information pointing to serious violations of international humanitarian law and international human rights law by Israel and by Palestinian armed groups. In some cases, these violations may amount to war crimes".

4. L'adhésion au Statut de Rome se fait par l'intermédiaire du Secrétaire général de l'ONU, qui agit en tant que dépositaire de ce traité.

5. Un examen préliminaire n'est pas une enquête mais un processus consistant à examiner les informations disponibles afin de déterminer en toute connaissance de cause, s'il existe une base raisonnable pour initier une enquête au regard des critères posés par le Statut de Rome. Le Procureur analysera en particulier les questions liées à la compétence, à la recevabilité et aux intérêts de la justice lorsqu'elle prendra sa décision, ainsi qu'il est prévu à l'article 53-1 du Statut de Rome. Le Bureau tient dûment compte de l'ensemble des observations et des points de vue qui lui sont transmis au cours de l'examen préliminaire, guidé exclusivement par les exigences du Statut de Rome pour exercer son mandat en toute indépendance et en toute impartialité. Le Statut de Rome n'impose aucun délai pour rendre une décision relative à un examen préliminaire. Le Bureau pourra décider, en fonction des faits et des circonstances propres à chaque situation, de continuer à recueillir des informations afin de rendre une décision dûment motivée en fait et en droit, d'ouvrir une enquête sous réserve, si nécessaire, d'une autorisation judiciaire, ou de ne pas en ouvrir.

6. Pour plus d'éléments sur ces critères, voir, D. Robinson, «Defining «Crimes Against Humanity » at the Rome Conference ", The American Journal of International Law, vol. 93, No. 1, Janvier 1999, pp. 43-57 ; ou encore, M. Delmas-Marty, I. Fouchard, E. Fronza, L. Neyret, Le crime contre l'humanité, Ed. Presses Universitaires de France, 2013, 128 p.

7. Breaking the Silence, Le livre noir de l'occupation israélienne : Les soldats racontent, Éditions Autrement, Paris, 2013, Témoignage $n^{\circ} 7$.

8. Idem., Témoignage $n^{\circ} 8$ « Je n'arrivais pas à croire que l'ordre de tuer puisse avoir été exécuté en une minute»; Témoignage $n^{\circ} 9$ "Peine de mort pour un homme désarmé »; Témoignage $n^{\circ} 10$ «Le commandant de bataillon a ordonné de tirer sur des gens qui essayaient de récupérer des corps »; Témoignage $\mathrm{n}^{\circ} 11$ « Il a descendu un garçon de onze ans »; etc. 
9. Voir, pour plus d'information sur les différents modes de participation à l'infraction, R. Prouvèze, "Les modes individuels de participation à l'infraction (action, coaction, complicité) 》, in H. Ascencio, E. Decaux et A. Pellet (dir.), Droit international pénal, Ed. Pedone, 2012, pp. 489-502.

10. TPIY, Chambre de première instance, Le Procureur c. Anto Furundzija, IT-95-17/1-T, Jugement, 10 décembre 1998: le TPIY a retenu que la complicité consiste en une aide, un encouragement, ou un soutien moral ayant un effet important sur la perpétration du crime.

11. Le Livre noir de l'occupation israélienne, Éditions Autrement, Paris, Octobre 2013, Préface «Les Briseurs de glace», par Zeev Sternhell (Professeur émérite de sciences politiques à l'université hébraïque de Jérusalem).

12. D. Lochak, "L'alerte éthique, entre dénonciation et désobéissance », AJDA, n 39, 24 novembre 2014, Dossier sur les Lanceurs d'alerte, pp. 2236-2241.

13. Assemblée parlementaire du Conseil de l'Europe, Résolution 1729 [2010], 29 avr. 2010, Protection des « donneurs d'alerte ».

14. K. Ambos, "The Right of Non-Self-Incrimination of Witnesses Before the ICC », LJIL, vol. 15, 2002, p. 173.

15. «Article $69 \quad$ Preuve

1. Avant de déposer, chaque témoin, conformément au Règlement de procédure et de preuve, prend l'engagement de lire la vérité. 2. Les témoins sont entendus en personne lors d'une audience, sous réserve des mesures prévues à l'article 68 ou dans le Règlement de procédure et de preuve. La Cour peut également autoriser un témoin à présenter une déposition orale ou un enregistrement vidéo ou audio, et à présenter des documents ou des transcriptions écrites, sous réserve des dispositions du présent Statut et conformément au Règlement de procédure et de preuve. Ces mesures ne doivent être ni préjudiciables ni contraires aux droits de la défense. 3. Les parties peuvent présenter des éléments de preuve pertinents pour l'affaire, conformément à l'article 64. La Cour a le pouvoir de demander la présentation de tous les éléments de preuve qu'elle juge nécessaires à la manifestation de la vérité. 4. La Cour peut se prononcer sur la pertinence et l'admissibilité de tout élément de preuve conformément au Règlement de procédure et de preuve, en tenant compte notamment de la valeur probante de cet élément de preuve et de la possibilité qu'il nuise à l'équité du procès ou à une évaluation équitable de la déposition d'un témoin ».

16. Fouchard, "Chapitre 25 - Outrage au tribunal », in Ascensio, Decaux, Pellet, (dir.), Droit international pénal, Ed. Pedone, Paris, 2012, p. 250.

17. CPI, Chambre de première instance V(b), Le Procureur c. Uhuru Muigai Kenyatta, Decision on the withdrawal of charges against Mr Kenyatta, 13 mars 2015, ICC-01/09-02/11-1005, https:// www.icc-cpi.int/CourtRecords/CR2015_02842.PDF.

18. ICC-01/09-01/11-2027-AnxI, Dissenting Opinion of Judge Herrera Carbuccia, https://www.icccpi.int/RelatedRecords/CR2016_02619.pdf.

19. CPI, Chambre de première instance V (a), Le Procureur c. William Samoei Ruto and Joshua Arap Sang, Public redacted version of Decision on Defence Applications for Judgments of Acquittal, 16 juin 2016, ICC-01/09-01/11-2027-Red-Corr, https://www.icc-cpi.int/CourtRecords/ CR2016_04384.PDF.

20. Pacte international relatif aux droits civils et politiques, Adopté et ouvert à la signature, à la ratification et à l'adhésion par l'Assemblée générale dans sa résolution 2200 A (XXI) du 16 décembre 1966, Article 14 3) g).

21. G. Mabanga, «Le rôle du conseil dans le cadre des témoignages incriminant leur auteur. Le cas de la Cour pénale internationale », La Revue des Droits de l'Homme, n 3, juin 2013.

22. Ch. 1re inst. I, 28 janvier 2009, le Procureur c/ Thomas Lubanga Dyilo, Transcription d'audience, ICC-01/04-01/06-T-110-Red3-FRA CT WT, p. 4, 1. 20-23. V., à ce sujet, S. Shoamanesh et 
A. Mbaye, "Article 55. Droits des personnes dans le cadre d'une enquête », in J. Fernandez et X. Pacreau (dir.), Commentaire article par article du Statut de Rome de la CPI, t. II, Pedone, Paris, 2012, p. 1265.

\section{RÉSUMÉS}

Cette contribution se concentre sur les témoignages divulgués par le groupe militant israélien "Breaking the Silence », relatant des exactions commises en territoires palestiniens occupés par des soldats de l'armée israélienne. L'article démontre que les actes en cause sont susceptibles de relever de la compétence de la Cour pénale internationale. Les auteurs des témoignages étant également auteurs des actes en cause, divulguer ces informations constitue pour eux une prise de risque de se voir accusés, poursuivis et jugés. Après avoir testé et établi la possibilité de qualifier les auteurs des témoignages de lanceurs d'alerte, nous partirons à la recherche d'un régime protecteur de ces soldats témoins et, par là même, des éléments d'information qu'ils prennent le risque de divulguer au bénéfice du travail de la Cour pénale internationale. Sera étudiée, puis affirmée la pertinence du droit de ne pas s'auto-incriminer, prévu par l'article $68 \mathrm{du}$ Statut de Rome, pour faire office de régime protecteur des soldats lanceurs d'alerte. Seront toutefois soulignées les imperfections du régime mis en place par le Statut de Rome pour répondre aux exigences de protection des auteurs de ces précieux témoignages.

This contribution focuses on the evidence disclosed by the Israeli activist group "Breaking the Silence" relating abuses in the Palestinian territories occupied by soldiers of the Israeli army. This contribution demonstrates that the acts in question are capable of falling within the jurisdiction of the International Criminal Court. The authors of the testimonies being also involved perpetrators, disclosing such information constitutes for them a risk of being charged, prosecuted and tried. Having tested and established the possibility of describing the perpetrators of testimonies of whistleblowers, we will go in search of a protective regime of such witness soldiers and, thereby, of the information they take the risk to disclose on the benefit of the work of the international criminal Court. The relevance of the right not to incriminate themselves, under Article 68 of the Rome Statute, to make protective regime of soldiers whistleblowers, will be studied and affirmed. However, the shortcomings of the regime established by the Rome Statute to meet the requirements of the protection of perpetrators of these precious testimonies will be highlighted.

\section{INDEX}

Mots-clés : lanceur d'alerte, droit pénal international, cour pénale internationale, Israël, Palestine, témoins, crime de guerre, crime contre l'humanité, auto-incrimination, statut de Rome, protection des lanceurs d'alerte, article 68 du statut de Rome, article 55 du statut de Rome, article $14 \mathrm{du}$ Pacte international relatif aux droits civils et politiques.

Keywords : whistleblower, international criminal law, international criminal court, Israel, Palestine, witnesses, war crime, crimes against humanity, self-incrimination, Rome statute, protection of whistleblowers, article 68 of the Rome statute, article 55 of the Rome statute, article 14 of the international covenant on civil and political rights. 
AUTEUR

REBECCA MIGNOT-MAHDAVI

Doctorante à l'European University Institute, Florence (Italie) 